\title{
Durability of First-Line Antiretroviral Treatment Regimens in a Cohort of HIV-Infected Patients
}

Perez Stachowski $\mathrm{J}^{1 *}$, Gonzalez-Domenech $\mathrm{CM}^{2}$, García Vallecillos $\mathrm{C}^{3}$, Perez-Camacho ${ }^{4}$, Vinuesa Garcia $\mathrm{D}^{5}$, Omar $\mathbf{M}^{6}$, Olalla $\mathrm{J}^{1}$, Palacios $\mathrm{R}^{2}$, Del Arco $\mathrm{A}^{1}$, Hidalgo-Tenorio $\mathrm{C}^{3}$, Santos $\mathrm{J}^{2}$, Prada $\mathrm{JL}^{1}$ and De la Torre $\mathrm{J}^{1}$

${ }^{1}$ Department of Infectious Diseases, Hospital Costa del Sol, Marbella, Spain

${ }^{2}$ Department of Infectious Diseases, Hospital Virgen de la Victoria, Málaga, Spain

${ }^{3}$ Department of Infectious Diseases, Hospital Virgen de las Nieves, Granada, Spain

${ }^{4}$ Department of Infectious Diseases, Hospital de Poniente, Almeria, Spain

${ }^{5}$ Department of Infectious Diseases, Hospital San Cecilio, Granada, Spain

${ }^{6}$ Department of Infectious Diseases, Complejo Hospitalario de Jaén, Jaén, Spain

\begin{abstract}
Aims: In recent years, increasing numbers of studies have been made of the durability of antiretroviral treatments (ART) and reasons for changing this medication, but few have evaluated the most recent therapeutic approaches. In this paper, we analyse the durability of the first-line ART regimen currently employed for patients infected with human immunodeficiency virus (HIV), and examine the reasons for treatment modification.
\end{abstract}

Method: A retrospective multicentre observational study was conducted of patients with HIV infection who started first-line ART between January 2012 and December 2013 in the provinces of Málaga, Granada, Jaén and Almería (Spain). The main study variable is the durability of the first-line ART regimen until its modification, the reasons for which are described. Survival analysis was performed using Kaplan-Meier curves to evaluate durability, and a Cox multiple regression model was constructed to identify associated factors.

Results: A total of 664 patients started first-line ART. The average durability was 20 months (95\% Cl: 17-22); at one year, $29 \%$ had abandoned this regimen, while $57 \%$ maintained it until the end of follow-up. The greatest durability was achieved with regimens based on rilpivirine (RPV), darunavir (DRV) and raltegravir (RAL). The main reason for change was toxicity $(20 \%)$, mainly gastrointestinal, associated with the use of protease inhibitors $(\mathrm{PI})$, and neuropsychiatric, associated with the use of efavirenz (EFV). HCV coinfection, AIDS diagnosis and type of ART regimen were all associated with reduced durability.

Conclusion: With the regimens currently applied, the durability of first-line ART is 20 months. At one year of treatment, this first-line regimen had been modified for $29 \%$ of patients, with toxicity being the main reason for change. Regimens based on RPV, DRV and RAL present greater durability, mainly due to their lower toxicity.

Keywords: Naive; Antiretroviral; Durability; Toxicity; HIV

\section{Introduction}

Antiretroviral Treatments (ART) have changed the course and prognosis of HIV infection. The emergence of combination therapy - new treatments based on families of drugs such as Integrase Inhibitors (INI) or the new Protease Inhibitors (PIs), with improved dosage parameters and increased tolerance - have radically changed the outlook in antiretroviral therapy by facilitating treatment compliance and reducing toxicity. All current treatment regimens of choice achieve high virological efficacy, thus providing a paradigm shift in which the discussion about which ART should best be chosen now focuses on the questions of tolerance/toxicity and convenience. Both of these issues can be measured in the durability or persistence of the first-line ART regimen, a parameter that reflects the potency, tolerance and comfort of the treatment received. In this paper we evaluate a real-life study of the durability of first-line ART regimens currently employed, and identify the reasons for their interruption.

\section{Material and Method}

\section{Study design}

This multicentre retrospective observational study was made of adult patients with HIV infection who started ART in hospitals in the south east of Spain. Inclusion criteria were diagnosis of HIV infection, aged at least 18 years and first-line ART regimen with triple therapy initiated between 1 January 2012 and 31 December 2013. Follow-up was performed until 30 June 2014.
The main study variable was the durability of treatment, defined as the time elapsed from the start of the first-line ART regimen to its discontinuation. The secondary study goal was to identify the main reasons for treatment interruption.

\section{Statistical analysis}

A survival analysis was performed until the occurrence of each of the outcome variables, represented by Kaplan-Meier survival curves. Cox proportional hazards regression models were constructed for independent variables, and multivariate analysis by Cox's regression model was performed to adjust for possible confounders, to identify interactions and to address time-dependent variables. The variables included in the multivariate model were sex, age, HCV infection at any

*Corresponding author: J Perez-Stachowski, Department of Infectious Diseases, Hospital Costa del Sol, Marbella Urbanizacion Sierrezuela Hill Club, calle Perejil 7, 29651, Mijas-Costa, Malaga, Spain; Tel: 0034626157354; E-mail: xpstachowski@gmail.com

Received July 23, 2018; Accepted August 02, 2018; Published August 09, 2018

Citation: Perez Stachowski J, Gonzalez-Domenech CM, García Vallecillos C, Perez-Camacho I, Vinuesa Garcia D, et al. (2018) Durability of First-Line Antiretroviral Treatment Regimens in a Cohort of HIV-Infected Patients. J AIDS Clin Res 9: 773. doi: 10.4172/2155-6113.1000773

Copyright: () 2018 Perez Stachowski J, et al. This is an open-access article distributed under the terms of the Creative Commons Attribution License, which permits unrestricted use, distribution, and reproduction in any medium, provided the original author and source are credited. 
moment, HIV transmission mechanism, current AIDS diagnosis and ART type.

\section{Results}

Six hundred and sixty-four patients began treatment (662 patientsyears follow-up). Of these, $86 \%$ were male, with an average age of 37 years (IQR 30-44). The most commonly used drug combinations were TDF/FTC/EFV (Tenofovir + Emtricitabine + Efavirenz) $243(36 \%)$ TDF/FTC/DRV (Tenofovir + Emtricitabine + Darunavir) $85(12 \%)$, TDF/FTC/RPV (Tenofovir + Emtricitabine + Rilpivirina) $82(12 \%)$, TDF/FTC/ATV (Tenofovir + Emtricitabine + Atazanavir) $50(7 \%)$, TDF/FTC/RAL (Tenofovir + Emtricitabine + Raltegravir) 46 (7\%), TDF/FTC/LPV (Tenofovir + Emtricitabine + Lopinavir) $33(5 \%)$ and others 125 (18\%). Of these, 81 (12\%) included ABC (Abacavir).

The first ART regimen was continued until the end of followup by $57 \%$ of the patients. The average treatment duration was 20 months (95\% CI: 17-22). At one year after starting, $29 \%$ of patients had interrupted treatment, and at two years, this figure had risen to $41 \%$. The overall rate of treatment interruption was 43.4 per 100 patients per year of treatment. The average durability, in months and by treatment combination was: TDF/FTC/EFV 21 (IC95\% 18-23), TDF/FTC/DRV 22 (IC95\% 14-29), TDF/FTC/RPV 21 (95\% CI: 19-21), TDF/FTC/ATV 15 (95\% CI: 11-18), TDF/FTC/RAL 21 (95\% CI: 19-23), TDF/FTC/LPV 10 (95\% CI: 5-14), others 18 (95\% CI: 14-21).

Treatment was interrupted by 287 patients (43\%), for the following main reasons: 1) ART-related toxicity, which affected 135 patients (20\%); 2) loss of follow-up, involving 61 patients (9\%); 3) changes for comfort/convenience, by 43 patients $(6 \%)$; 4) virological failure, in 16 patients $(2 \%)$; 5) voluntary treatment abandonment, by 11 patients $(1.7 \%) ; 6)$ changes to prevent interactions, in 9 patients $(1.4 \%) ; 7)$ death, 6 patients $(1 \%) ; 8$ ) others, 6 patients (1\%). Interruptions due to toxicity can be seen in Figure 1. According to the multivariate analysis, the variables that were significantly associated with less durability were HCV coinfection, AIDS diagnosis and the ART regimen applied.

\section{Discussion}

In this study of 664 HIV-infected patients who started first-line
ART, the average durability was 20 months (95\% CI: 17-22), with a rate of treatment modification of $29 \%$ per year, while $57 \%$ of patients maintained the original regimen until the end of follow up. The best results in terms of durability were obtained by regimens based on RPV, DRV and RAL. The main reason for change was toxicity $(20 \%)$, especially digestive toxicity, which was associated with the use of PIs, and neuropsychiatric, associated with EFV.

In the ICONA cohort [1], 26\% of patients had discontinued treatment within the first year, the main reasons being simplification (changes to drugs which can be administered once a day), followed by intolerance and toxicity. The percentage of treatment interruptions was similar to that observed in our study, although there was a greater percentage of changes due to simplification. This may be due to the fact that the inclusion period for the Italian cohort was earlier (2008-2014) and therefore ART was started with more complex and expensive regimens, which favoured subsequent simplification. In the Swiss cohort [2], which included 1318 patients who started ART in the period 2005-2008, there were $29 \%$ treatment interruptions per year, with toxicity being the most common cause (14\%). In the UK study [3], carried out from 2012 to 2015 , with a median follow-up period and type of ART similar to ours, the rate of treatment interruption was identical to ours (43 interruptions per 100 patients/year), with toxicity being the main reason. It should be noted that, compared to two studies of similar characteristics carried out at earlier times in several centres that also participated in the present study, our results were better than those obtained in the earlier study [4] (1997-2003, 36\% treatment interruptions/year) and similar to those of the more recent one [5] (2007-2010, 28\% treatment interruptions/year). Virological failure was the reason for change in only $2 \%$ of cases, which confirms the efficacy of the results obtained in the pivotal studies with RPV [6], DRV [7] and RAL [8].

By type of ART, PIs have traditionally had poorer tolerability than nucleoside analogue reverse transcriptase inhibitors (NRTIs), which thus have a higher rate of treatment interruption. However, this difference has been neutralised with the introduction of DRV as a treatment option [7]. With respect to Integrase Inhibitors (INI), few cohort studies have been undertaken (of which most are focused on RAL), but this approach is known to produce greater durability

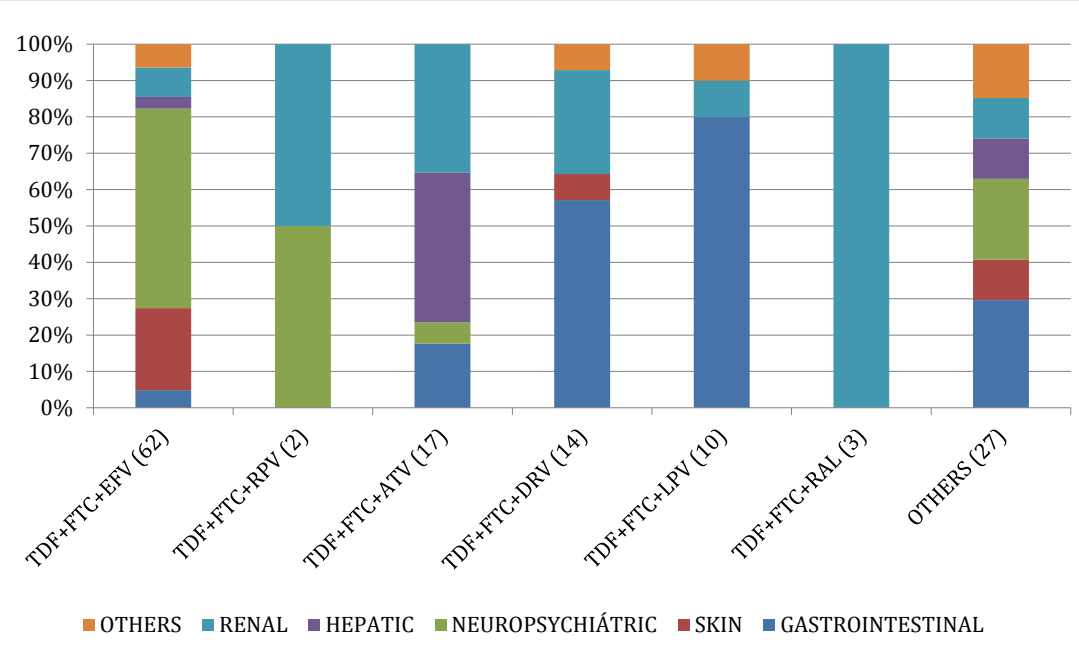

TDF/FTC/EFV: Tenofovir+Emtricitabine+Efavirenz. TDF/FTC/RPV: Tenofovir+Emtricitabine+Rilpivirina. TDF/FTC/ATV: Tenofovir+Emtricitabine+Atazanavir. TDF/ FTC/DRV; Tenofovir+Emtricitabine+Darunavir. TDF/FTC/LPV: Tenofovir+Emtricitabine+Lopinavir. TDF/FTC/RAL: Tenofovir+Emtricitabine+Raltegravir.

Figure 1: Toxicity by type of ART regimen. 
than other regimens [9]. These results show that older PIs produce more toxicity than NRTIs and therefore higher rates of treatment interruption, but studies of more recent ones, such as DRV, and of INI, report better results than are achieved by EFV, as in our own analysis. RPV also has very good tolerability, with few interruptions due to toxicity, although there may be a selection bias since, according to the clinical guidelines, this regimen should be initiated in patients with a viral load $<100,000$ copies and CD4 $>200$. In our study, PIs were used for patients who started from a worse immunological situation, and so there was a greater risk of interactions with other drugs or of virological failure, which is in accordance with the findings of other studies.

According to the previous studies considered, the factors traditionally associated with a higher rate of treatment interruption are female gender, younger age, AIDS diagnosis, PI-containing regimens, and greater numbers of pills in the regimen.

Our study present the following limitations: 1) We did not include patients treated with one or two-drug regimens, since this practice is not common in our hospital unit and the number of cases is too low to allow reliable conclusions to be drawn; 2) The data were compiled from the digital medical history and treatment modifications were made according to the judgment of the clinician responsible, making it difficult to characterise the side effects. For example, the definition of renal toxicity includes both increased creatinine levels and the appearance of proteinuria. A prospective study would help overcome this limitation; 3) Our study did not include new, currently front-line, antiretrovirals such as dolutegravir or elvitegravir because at the date of data compilation they were not available; 4) Despite it being a factor associated with durability, given the retrospective nature of the study, compliance was neither measured nor estimated. Again, carrying out a prospective study would remove this limitation, but this factor does not affect the results obtained or the differences found between the drugs.

\section{Conclusions}

Despite the appearance of new molecules, the rate of treatment interruption within one year of starting treatment remains high (29\%). At present, treatment toxicity is the major cause of this change (affecting $20 \%$ of patients starting ART). Regimens based on RPV, DRV and RTG have greater durability, which is associated with their lower toxicity.

\section{References}

1. Di Biagio A, Cozzi-Lepri A, Prinapori R, Angarano G, Gori A, et al. (2016) Discontinuation of antiretroviral therapy in clinical practice: Moving toward individualized therapy. J Acquir Immune Defic Syndr 71: 263-271.

2. Elzi L, Marzolini C, Furrer H, Ledergerber B, Cavassini M, et al. (2010) Treatment modification in HIV-infected individuals starting combination antiretroviral therapy between 2005 and 2008: Swiss HIV cohort study. Arch Intern Med 170: 57-65.

3. Lewis JM, Smith C, Torkington A, Davies C, Ahmad S, et al. (2017) Realworld persistence with antiretroviral therapy for HIV in the United Kingdom: A multicentre retrospective cohort study. J Infect 74: 401-407.

4. De la Torre-Lima J, Santos J, Perea-Milla E, Pérez I, Moreno F, et al. (2008) First regimen of antiretroviral treatment in patients with HIV infection: Durability and factors associated with its modification. Enferm Infecc Microbiol Clin 26 : 416-422.

5. De La Torre-Lima J, Aguilar A, Santos J, Jiménez-Oñate $F$, Marcos $M$, et al. (2014) Durability of the first antiretroviral treatment regimen and reasons for change in patients with HIV infection. HIV Clin Trials 15: 27-35

6. Cohen CJ, Molina JM, Cahn P, Clotet B, Fourie J, et al. (2012) Efficacy and safety of rilpivirine (TMC278) versus efavirenz at 48 weeks in treatmentnaive HIV-1-infected patients: Pooled results from the phase 3 double-blind randomized ECHO and THRIVE Trials. J Acquir Immune Defic Syndr 60: 3342.

7. Orkin C, DeJesus E, Khanlou H, Stoehr A, Supparatpinyo K, et al. (2013) Final 192-week efficacy and safety of once-daily darunavir/ritonavir compared with lopinavir/ritonavir in HIV-1-infected treatment-naïve patients in the ARTEMIS trial. HIV Med 14: 49-59.

8. Lennox JL, Dejesus E, Berger DS, Lazzarin A, Pollard RB, et al. (2010) Raltegravir versus Efavirenz regimens in treatment-naive HIV-1-infected patients: 96-week efficacy, durability, subgroup, safety, and metabolic analyses. J Acquir Immune Defic Syndr 55: 39-48.

9. Eaton E, Tamhane A, Burkholder G, Willig J, Saag M, et al. (2016) Unanticipated effects of new drug availability on antiretroviral durability: Implications for comparative effectiveness research. Open Forum Infect Dis 3: ofw109. 